\title{
Progressions
}

\section{A Search for a Cortical Map of Auditory Space}

\author{
ㄱotohn C. Middlebrooks ${ }^{1,2,3,4}$ \\ ${ }^{1}$ Department of Otolaryngology, ${ }^{2}$ Department of Neurobiology and Behavior, ${ }^{3}$ Department of Cognitive Sciences, and ${ }^{4}$ Department of Biomedical \\ Engineering, University of California at Irvine, Irvine, California 92697-5310
}

This is the story of a search for a cortical map of auditory space. The search began with a study that was reported in the first issue of The Journal of Neuroscience (Middlebrooks and Pettigrew, 1981). That paper described some unexpected features of spatial sensitivity in the auditory cortex while failing to demonstrate the expected map. In the ensuing 40 years, we have encountered the following: panoramic spatial coding by single neurons; a rich variety of response patterns that are unmasked in the absence of general anesthesia; sharpening of spatial sensitivity when an animal is engaged in a listening task; and reorganization of spatial sensitivity in the presence of competing sounds. We have not encountered a map, but not through lack of trying. On the basis of years of negative results by our group and others, and positive results that are inconsistent with static point-to-point topography, we are confident in concluding that there just ain't no map. Instead, we have come to appreciate the highly dynamic spatial properties of cortical neurons, which serve the needs of listeners in a changing sonic environment.

\section{Introduction}

Maps are ubiquitous in the sensory cortex. Examples include topographic representations of visual space in the visual cortex, of the body surface in somatosensory cortex, and of sound frequency in the auditory cortex. In hearing, location in space is a prominent attribute of a sound source, and it seems reasonable to search for point-to-point maps of the locations of sound sources in the world onto locations in the auditory cortex. In support of that quest are observations that unilateral lesions or inactivation of auditory cortex can disrupt performance of operant tasks requiring localization of contralateral sounds (Jenkins and Merzenich, 1984; Malhotra et al., 2004), which demonstrate the necessity of cortical function for spatial hearing. Also, auditory space maps are found in subcortical structures (Knudsen and Konishi, 1978; Middlebrooks and Knudsen, 1984), which demonstrates the feasibility of topographic spatial representation in the brain. Here, I describe a 40 year search for a cortical map of auditory space that was begun with work that was reported in the first issue of The Journal of Neuroscience.

\section{A search for a cortical map of auditory space}

My dissertation work in Michael Merzenich's laboratory at the University of California San Francisco involved the functional organization of the auditory cortex with emphasis on binaural hearing. Merzenich and others had demonstrated that one

\footnotetext{
Received Mar. 9, 2021; revised May 10, 2021; accepted May 12, 2021.

This work (my spatial-hearing research) was supported by National Institutes of Health R01 DC000420. I thank my mentors and trainees with whom I have had the pleasure of doing this work. Preparation of this article brought to mind fond memories of working with Jack Pettigrew who, sadly, died after a car accident in 2019. I also thank long-time assistant Zekiye Onsan for managing the laboratory and collecting human psychophysical data; and long-time assistant Beth McGuire for herding cats.

The author declares no competing financial interests.

Correspondence should be addressed to John C. Middlebrooks at j.midd@uci.edu.

https://doi.org/10.1523/JNEUROSCI.0501-21.2021

Copyright $\odot 2021$ the author
}

dimension of the auditory cortex is devoted to a map of sound frequency, corresponding to the frequency organization of the cochlea (Merzenich et al., 1975). Early in my project, I showed in cats that the cortical dimension perpendicular to the frequency map (along isofrequency contours) contains repeating modules of neurons showing either excitatory or inhibitory binaural interactions (Middlebrooks et al., 1980; also described by Imig and Adrian, 1977; Imig and Brugge, 1978). Auditory spatial sensitivity is derived primarily from binaural interactions. For that reason, we began thinking of an auditory space map in the cortex.

Shortly before that time, Knudsen and Konishi (1978) at Caltech had discovered a detailed auditory space map in the midbrain of the barn owl. They used an anechoic chamber that they had equipped with a mechanism for varying the position of a sound source around an animal. It seemed so obvious to me that there must be a space map in the cat's auditory cortex that I hatched the idea of visiting Konishi's laboratory and picking what seemed like low-hanging fruit. I wrote to Konishi proposing such a plan, and he generously agreed to let me test my hypothesis in his laboratory. Key to his agreement, I think, was that Jack Pettigrew was also on the faculty at Caltech at that time and was interested in gaining more exposure to auditory neuroscience. Pettigrew agreed to supervise me.

Pettigrew and I recorded from neurons in the primary auditory cortex (area A1) of anesthetized cats. Our experimental design involved first finding the characteristic frequency $(\mathrm{CF})$ to which each neuron was tuned. Pure tones at each neuron's CF, then, were used to delimit the spatial receptive field within which a sound would elicit above-background activity of the neuron. Sound levels generally were set to $10 \mathrm{~dB}$ above each neuron's threshold.

By analogy with the owl midbrain, we hypothesized that cortical neurons in cats would have restricted receptive fields, and that such fields would vary systematically as a function of cortical location. Our results were quite contrary to the hypothesis. 

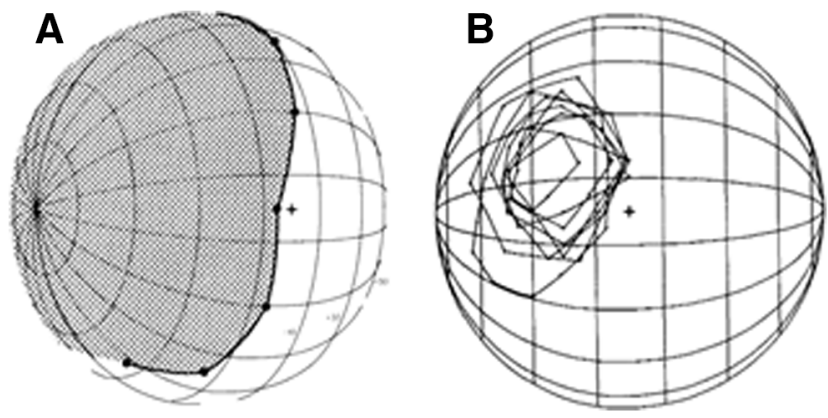

Figure 1. Spatial receptive fields. $\boldsymbol{A}$, The receptive field of a hemifield neuron is represented by shading. The view is toward the cat, from $45^{\circ}$ to the right of the frontal midline. $\boldsymbol{B}$, Receptive fields of 8 axial neurons recorded in 1 cat. The viewpoint is from the frontal midline. Reprinted from Middlebrooks and Pettigrew (1981).

About half of the neurons that we encountered responded to sounds presented from anywhere around the animal. We named these omnidirectional neurons. Of the other half of neurons, those with $\mathrm{CFs}<12 \mathrm{kHz}$ had receptive fields bordered near the frontal midline, with the field extending to behind the animal on the contralateral side; those were named hemifield neurons (Fig. $1 A)$. Location-sensitive neurons with $\mathrm{CFs}>12 \mathrm{kHz}$ had more-restricted receptive fields that were bordered entirely within the frontal hemifield. In each cat, however, all such fields tended to be located concentrically around what turned out to be the acoustical axis of the contralateral external ear (the pinna). The locations of those receptive fields varied among cats, probably because of differences in positioning of the pinna, and the fields could be shifted by physically moving the pinna. We named these axial neurons (Fig. $1 B$ ). Notably, what we did not find was a map of auditory space.

We prepared to write up the results. Pettigrew had gotten wind of a new journal to be offered by the Society of Neuroscience, and he urged me to submit the work there. I was uneasy about taking a chance on the success of an unproven journal. Pettigrew persisted. We submitted the manuscript to The Journal of Neuroscience, and it was published in the first issue (Middlebrooks and Pettigrew, 1981).

Our failure to find a space map in the auditory cortex was disappointing to us and was not exactly cheered within the auditory research community. At that time, most high-quality experimental designs called for resection of the external ear (the pinna) to provide a well-calibrated path to the tympanic membrane. For that reason, our use of a preparation with intact pinnae was scorned by some, and our demonstration of axial units was greeted as "just a pinna effect." In the next few years, however, many people grew to accept that the pinna is a critical component of spatial hearing. That realization led to a brief flurry of studies of directionality of the cat's pinna (e.g., Middlebrooks and Pettigrew, 1981; Phillips et al., 1982; Calford and Pettigrew, 1984; Middlebrooks and Knudsen, 1987; Musicant et al., 1990; Rice et al., 1992).

In retrospect, there were a number of things that we could have done better in our 1981 study had we given more thought to known properties of spatial hearing. First, we used pure-tone stimuli. That seemed to make sense for study of area A1, in which neurons respond well to tones and show sharp frequency tuning. Localization of pure tones in real-world situations, however, can be problematic because tones do not permit use of the spectral-shape cues that are provided by the direction-dependent interaction of broadband sound with the head and external ears (e.g., Middlebrooks and Green, 1991). Broadband noise bursts

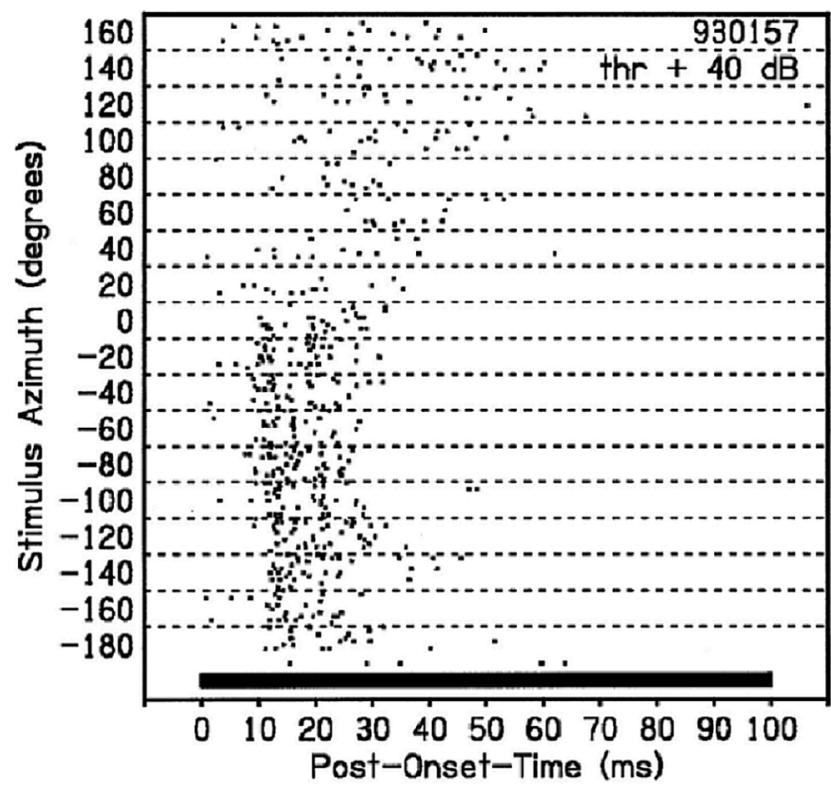

Figure 2. Location-specific temporal firing patterns of a cortical neuron. This raster plot represents responses of a single neuron in the primary auditory cortex (area A1). Each horizontal row of dots represents one train of action potentials. Responses varied in timing and magnitude as a function of sound-source location in azimuth (shown on the vertical axis). Eight trials are represented for each azimuth. Bottom, Bar represents the duration of the 100 ms broadband stimuli. Reprinted from Middlebrooks et al. (1998).

would have been a better choice. Second, we tested sounds primarily at levels only $10 \mathrm{~dB}$ above each neuron's threshold. We did that because we found receptive fields to be more compact at near-threshold sound levels. In human psychophysics, however, localization is degraded at near-threshold levels, improving with increasing sound level toward an asymptote at $\sim 20 \mathrm{~dB}$ above threshold and remaining accurate to at least $60 \mathrm{~dB}$ above threshold (e.g., Sabin et al., 2005). Our use of near-threshold sound levels probably exaggerated the "pinna effect" seen in axial neurons. Third, we defined a neuron's receptive field as the entire region within which a sound elicited any above-background response. Later work has shown that, within such a receptive field, neural spike rates vary widely. We should have quantified neural spike rates within receptive fields.

Nine years passed. In 1990, two groups reported new studies of spatial sensitivity in the cat auditory cortex (Imig et al., 1990; Rajan et al., 1990a,b). Both groups used broadband noise bursts. The noise bursts elicited a greater variety of spatial tuning than we had observed using CF tones, but any particular neuron generally showed the same class of spatial tuning when tested with tones or noise (Rajan et al., 1990b). The percentage of omnidirectional neurons was lower than we observed. That almost certainly reflected the use of higher spike-rate criteria for defining receptive fields in the later studies. Both groups studied the effects of varying sound level. Generally, receptive fields tended to increase in size with increasing sound level, as we had illustrated for two examples in our 1981 paper. The Imig group found that neurons having nonmonotonic rate/level functions were more likely to show spatially restricted receptive fields. Both groups measured spike rates as a function of sound location and showed that the locations that elicited peak activity varied rather widely among neurons throughout the frontal half of space. Consistent with our results, neurons having similar receptive field types tended to cluster together in the cortex, and clusters of a similar type could be found at multiple distinct sites 


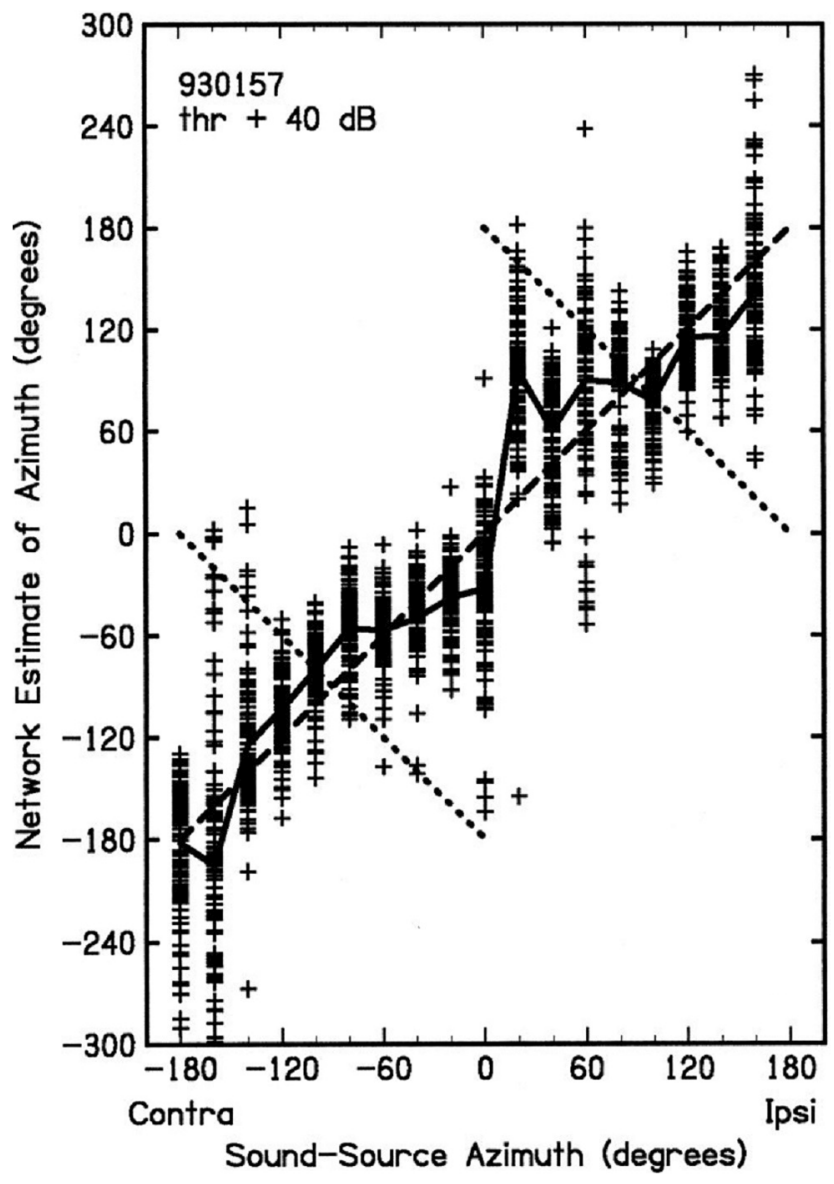

Figure 3. Estimates of sound-source locations based on spike patterns from the neuron represented in Figure 2. The estimated locations (vertical axis) are plotted as a function of actual stimulus locations (horizontal axis). Solid curve indicates the median estimate of each source location. The estimates tend to cluster around the diagonal line with positive slope that represents correct localization. Dotted lines indicate loci of correct front/back confusions. Reprinted from Middlebrooks et al. (1998).

along a particular isofrequency contour. Again, a cortical map of auditory space was conspicuous by its absence.

\section{Panoramic spatial coding}

It was difficult to let go of the notion that there must be a cortical space map. I assumed that we were missing it either by using the wrong stimuli or by looking in the wrong place. In my first faculty position, I convinced myself and a National Institutes of Health review panel that the key was to use broadband stimuli at moderate sound levels and to explore cortical areas in which neurons show broad frequency tuning: the second auditory field (A2) and the anterior ectosylvian sulcus area. We soon found, however, that nearly all units in those cortical fields responded with at least $25 \%$ of their maximum rates for sound-source locations throughout $360^{\circ}$ of azimuth (i.e., throughout the horizontal dimension). That essentially doomed any notion of an auditory space map based on tightly bordered receptive fields. Also, peaks in plots of spike rate versus azimuth failed to show any consistent trend as a function of position in the cortex.

We were intrigued by raster plots that showed temporal spike patterns of single neurons elicited by various sound source locations; an example is shown in Figure 2. We had been representing neural responses by simple spike counts. Might we be missing out on important stimulus-related information carried
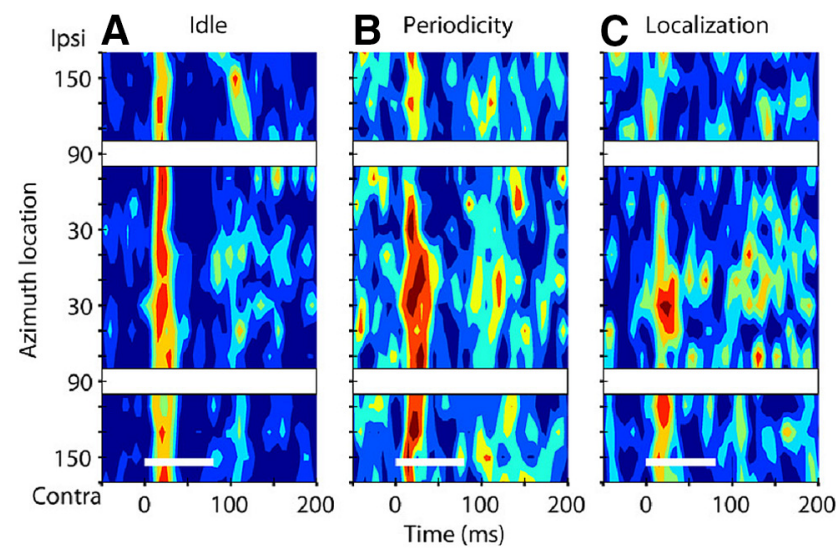

Figure 4. Location-specific response patterns in three task conditions. The three panels represent responses of the same neuron in Idle $(\boldsymbol{A})$, Periodicity $(\boldsymbol{B})$, and Localization $(\boldsymbol{C})$ task conditions, as described in the text. Each panel plots mean spike rates as colors as a function of time relative to stimulus onset (horizontal axis) and sound-source location in the horizontal plane (vertical axis). Bottom, Narrow white bar represents the 80 ms duration of the broadband stimulus. Contralateral and ipsilateral $90^{\circ}$ locations were not tested. Reprinted from Lee and Middlebrooks (2011).

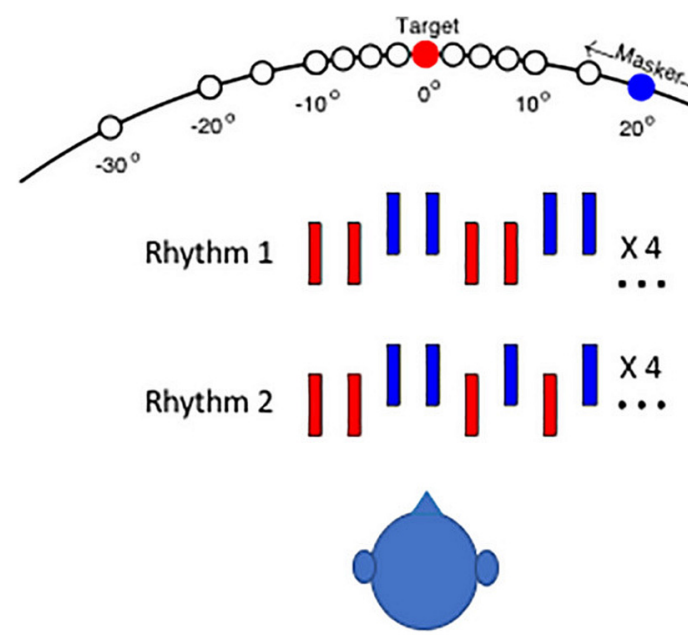

Figure 5. Experimental setup for the spatial stream segregation psychophysical task (Middlebrooks and Onsan, 2012). Top, Arc represents an array of loudspeakers positioned in the horizontal plane. In each session, the target source was fixed at the listener's $0^{\circ}$ or right $40^{\circ}$, and the masker source varied in location from trial to trial. Rows of colored bars represent rhythmic sequences of 20-ms-duration noise bursts: red from the target and blue from the competing masker. Each sequence of 8 target and masker bursts was repeated 4 times continuously. The listener's task on each trial was to report whether Rhythm 1 or Rhythm 2 was presented. That task was impossible when the target and masker source were colocated; but with sufficient target-masker separation, the target sequence popped out and the rhythm could be identified. Reprinted from Middlebrooks and Waters (2020).

by latencies and temporal density of neural spike patterns? We adapted an artificial neural network to estimate the locations of sound sources based on the location-dependent spike patterns of single neurons. Figure 3 shows estimates of sound-source locations based on spike patterns of the neuron represented in Figure 2. Location estimates tended to lie near the positive diagonal that indicated correct location identification. Rather than signaling only that a sound source was inside or outside of a particular best area (as in a labeled line model), the spike patterns of this neuron could signal, with more or less accuracy, locations throughout $360^{\circ}$ of azimuth. We referred to this as panoramic location coding (Middlebrooks et al., 1994, 1998). Viewed in this way, the broad spatial tuning of 

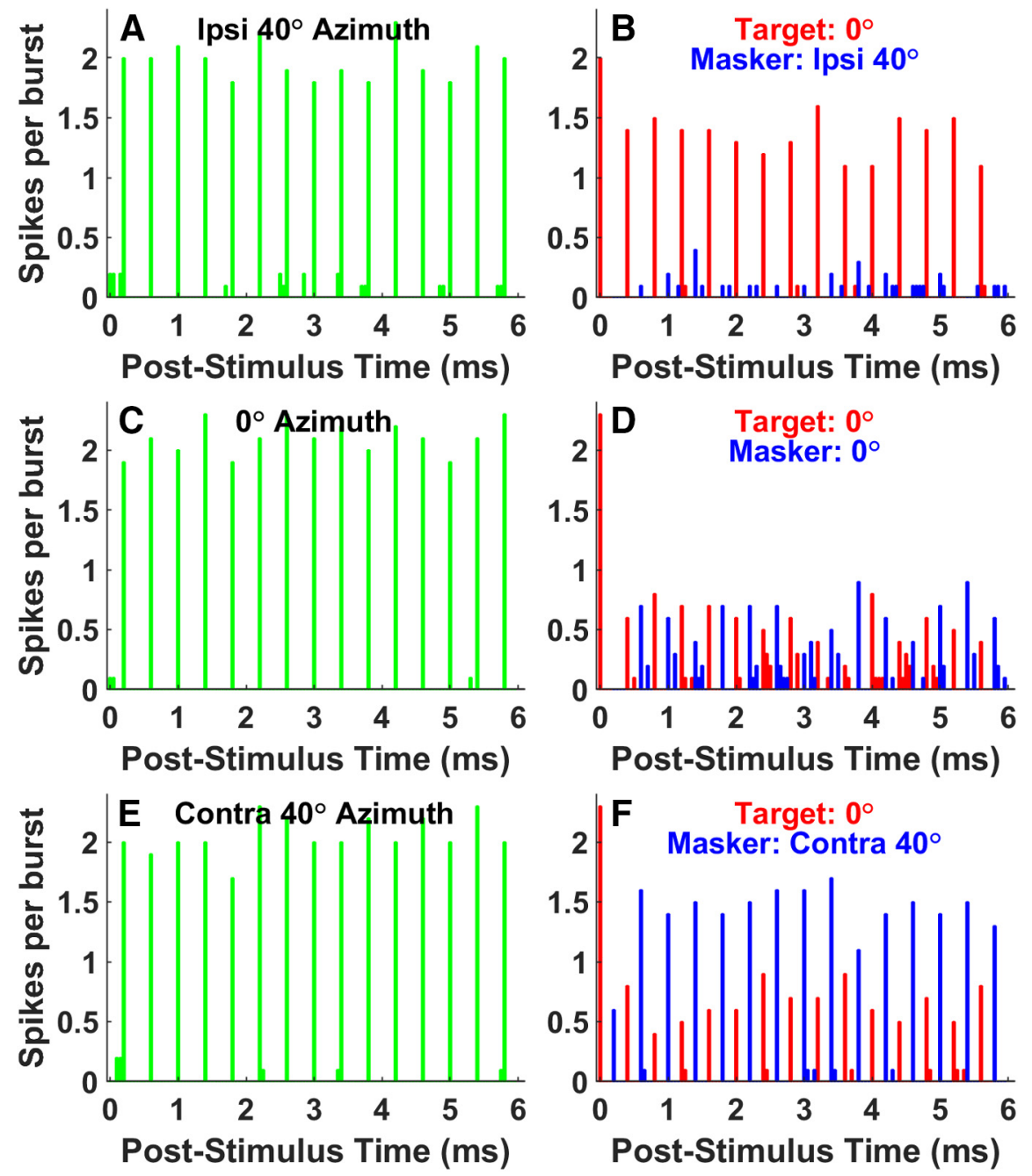

Figure 6. Post-stimulus time histograms representing mean spike counts synchronized to trains of 5 ms noise bursts. $\boldsymbol{A}, \boldsymbol{C}$, $\boldsymbol{E}$, Green bars represent responses to a single source at ipsilateral $40^{\circ}, 0^{\circ}$, or contralateral $40^{\circ}$, respectively, presented at a rate of $2.5 /$ s. $\boldsymbol{B}, \boldsymbol{D}, \boldsymbol{F}$, Responses synchronized to the target (red) fixed at $0^{\circ}$ and to the masker (blue) that was located at the three indicated directions. The aggregate rate of target and masker bursts in this example was $5 / \mathrm{s}$. Reprinted from Middlebrooks and Bremen (2013).

single neurons could be seen as a benefit rather than a liability. Neurons with such panoramic sensitivity were found throughout the cortical areas that we studied. That means that the location of any particular sound-source location is represented in a highly distributed code within and among cortical areas.

One lingering concern about our failure to observe a cortical map of auditory space was that all of our experiments had been conducted in conditions of general anesthesia. To address that issue, we began recording chronically from awake cats in cortical area A1 (Mickey and Middlebrooks, 2003). The results obtained in awake cats showed much more diverse spatial sensitivity than we had observed in the anesthetized preparation. We quantified the spatial tuning width of a neuron as the range of azimuths that elicited a half-maximal spike rate. Such tuning widths in the awake preparation ranged from $30^{\circ}$ to $360^{\circ}$ (median $150^{\circ}$ ). Tuning widths generally were less sensitive to increases in sound level than observed in anesthetized animals. As in the anesthetized condition, most neurons carried location-related information in the timing of spike patterns. Despite the differences in results obtained with or without anesthesia, the results from awake cats supported our observations of distributed representation of sound sources by panoramic neurons and further refuted our hypothesis that auditory space might be represented by a map in the auditory cortex.

\section{Rate coding and an opponent channel model}

The representation of sound-source locations by spike patterns that we observed can be thought of as a form of rate coding, generalized in our case by inclusion of temporal information. We and other groups have expanded on rate codes to involve the distribution of spike rates among populations of individual neurons or the summed activity within neural populations.

We estimated sound-source locations based on spike patterns drawn randomly from ensembles of neurons recorded from area A2 in anesthetized cats. Populations as large as 128 neurons yielded localization accuracy closely matching that of psychophysical sound localization by cats (May and Huang, 1996; Furukawa et al., 2000). Miller and Recanzone (2009) estimated sound locations based on firing rates of ensembles of 128 neurons in several cortical areas in alert macaques. Their results showed that near-midline locations are coded most accurately by neurons in area $\mathrm{R}$ and that far-contralateral and rear locations are coded most accurately by neurons in area CL. Werner-Reiss and Groh (2008) emphasized the monotonic increase in spike rate associated with increasing stimulus contralaterality in macaque auditory cortex and concluded that the "representational format" for sound-source location is a monotonic rate-versus-laterality code. Somewhat surprisingly, the summed population response in that study varied by only $\sim 8 \%$ across all frontal sound locations.

In anesthetized cats, the majority of spatially sensitive cortical neurons respond maximally to far contralateral locations, meaning that the function of spike rate versus sound-source location is steepest for near-midline locations. We tested an opponentchannel model in which sound-source locations were computed from the difference in total spike rates between contralaterally and ipsilaterally tuned populations of neurons (Stecker et al., 2005). That computation was quite successful in identifying the locations of sound sources within $20^{\circ}$ of the midline, although accuracy fell off rapidly for more eccentric locations. That the computation was based on the difference in activity between two neural populations made it fairly stable across changing sound levels.

The opponent-channel model has gained some favor in the literature, particularly in studies that provide little (e.g., electroencephalography) or no (psychophysics) resolution of brain anatomy (e.g., Phillips and Hall, 2005; Briley et al., 2013). Models that add a third, midline, channel are even more successful than the two-channel model (Dingle et al., 2010; Briley et al., 2016). 
In my present understanding, a two- or three-channel representation of sound-source location greatly devalues the wealth of information that is carried by the varied spatial tuning of single neurons. Another limitation of such fewchannel representations is that they rely on the balance of activity between a large population of contralaterally tuned neurons and a much smaller population of ipsilaterally tuned neurons within each cortical hemisphere; comparisons between cortical hemispheres cannot account for the observation that unilateral cortical inactivation results in only a contralateral localization deficit (Malhotra et al., 2004). Also, opponent models produce high acuity for near-midline sounds, but markedly degraded acuity for lateral locations, much more so than is observed psychophysically (e.g., Makous and Middlebrooks, 1990). Finally, it is difficult to reconcile a few-channel model with the cortical dynamics that are described in the next two sections.

\section{Spatial sensitivity modulated by task engagement}

Our earlier studies treated auditory spatial representation as static, with little or no influence of the behavioral state of the animal. Mickey and Middlebrooks (2003) observed some task dependence of responses in a small minority of neurons, but that was not a major focus of that study. We returned to the issue of task engagement (Lee and Middlebrooks, 2011, 2013). Cats were trained to initiate a trial by pressing and holding a pedal, which triggered a sequence of brief noise bursts from varying azimuths in the horizontal plan. The cat was rewarded for releasing the pedal when it heard a target sound. The target sound was a click train, in the Periodicity task, or a noise burst from a high elevation, in the Localization task. Cortical responses were compared across Periodicity, Localization, and off-task, Idle conditions.

Nearly half of neurons studied in area A1 sharpened their spatial tuning significantly when cats were engaged in the Periodicity task, and a smaller percentage showed further sharpening during the Localization compared with the Periodicity condition (Lee and Middlebrooks, 2011). An example of the most common pattern is shown in Figure 4. In the Idle condition, that neuron responded to the onset of the sound regardless of its location. The receptive field contracted to near-frontal and contralateral locations during the Periodicity task and sharpened further in the Localization task. The sharpening of tuning was accomplished by increased suppression in nonpreferred (typically, ipsilateral) sound locations, not by facilitation in preferred areas.

We compared the effects of task engagement on spatial sensitivity among neurons in area A1, the dorsal zone (DZ), and the posterior auditory field (PAF) (Lee and Middlebrooks, 2013), all of which are necessary for normal localization behavior (Malhotra et al., 2004, 2008). Approximately $30 \%$ of neurons in DZ and PAF showed a long-latency response that was more location-sensitive than the onset response. In PAF, the long-latency location-sensitive response tended to increase in magnitude during task performance. Areas DZ and PAF differed markedly from each in regard to rate coding by ensembles of 16 neurons. Area DZ showed accuracy approaching that of behaving cats for locations near the frontal midline. Location sensitivity of PAF units was essentially orthogonal to that of DZ, with greatest accuracy for extreme lateral locations. In both areas, estimates were most accurate in on-task conditions.

The studies in behaving cats demonstrated that spatial tuning of neurons could sharpen when the cat was engaged in a listening task, particularly when the task required localization. Nevertheless, there still was no evidence for spatial topography. Indeed, the demonstration that the spatial tuning of neurons was so dynamic argued against the notion of a static cortical space map.

\section{Spatial stream segregation}

An important function of spatial hearing is to aid in hearing out signals amid competing sounds. That is, spatial separation of target and masking sound sources can produce spatial release from masking. All along, I had thought that study of sound localization would teach me something about spatial release from masking. Eventually, I got around to studying spatial release directly. I soon learned that there are many situations in which spatial hearing provides relatively little release from masking, particularly in cases of energetic masking in which there is substantial overlap in the spectra and timing of the target and masker(s). Spatial hearing turns out to be quite important, however, in situations in which a listener must disentangle temporally interleaved sequences of sounds from multiple sources, as when a sequence of syllables from one talker must be disentangled from a sequence of syllables from a second talker. That is a problem of spatial stream segregation.

We began our study of the brain mechanisms of spatial stream segregation with psychophysics. Human listeners identified target sequences of broadband noise bursts having one of two rhythms, as represented by the red bars in Figure 5. Identification of Rhythm 1 versus Rhythm 2 was easy when the target sounds were presented alone but became challenging when noise bursts from another source were interleaved in time, as represented by the blue bars. By design, discrimination of the 
rhythms was impossible when the target and masker sources were co-located. When target and masker sources were separated in space, however, sequences were heard as distinct streams, and the target rhythm could be recognized. Human listeners could do the task with a target-masker separation as narrow as $8^{\circ}$ in azimuth (Middlebrooks and Onsan, 2012). In a feline version of the task, cats exhibited thresholds at $\sim 10^{\circ}$, nearly as acute as humans (Javier et al., 2016).

We sought a cortical mechanism for spatial stream segregation. Our previous measures of spatial receptive fields gave us no reason to expect spatial resolution in the cortex anything like what we had observed in the psychophysical experiments. Nevertheless, we tested neurons in area A1 in anesthetized cats with interleaved sequences of noise bursts from paired source locations, similar to the stream segregation stimuli (Middlebrooks and Bremen, 2013). We were delighted to discover responses like those represented in Figure 6. The left column of post-stimulus time histograms shows the responses of a single neuron in area A1 synchronized to sequences of $5 \mathrm{~ms}$ noise bursts. The neuron showed essentially no selectivity among source locations at ipsilateral $40^{\circ}, 0^{\circ}$, or contralateral $40^{\circ}$ (Fig. $6 A, C, E$, respectively). Prominent spatial sensitivity appeared, however, when an interleaved sequence of sound bursts was added, represented in the right column of histograms. Here, the red bars represent responses synchronized to a target source fixed at $0^{\circ}$, and the blue bars represent responses to a masker source at ipsilateral $40^{\circ}, 0^{\circ}$, or contralateral $40^{\circ}$ (Fig. $6 B, D, F$, respectively). When target and masker sources were co-located at $0^{\circ}$ (Fig. 6D), addition of the masker tended to suppress the ongoing response to both target and masker. When the masker source was moved to ipsilateral $40^{\circ}$, however, suppression of the response to the target (red bars) was released and the response to the masker (blue bars) was further suppressed (Fig. 6B). Conversely, when the masker source was moved to contralateral $40^{\circ}$ (Fig. $6 \mathrm{~F}$ ), the masker captured the response of the neuron and the response to the target was suppressed. Neurons that synchronized preferentially to the more contralateral of a pair of sound sources (e.g., the example in Fig. 6) tended to form cortical modules distinct from those that favored the more ipsilateral source.

Addition of a competing sound source clearly sharpened the stimulus-synchronized spatial sensitivity of the neuron represented in Figure 6, and that was a common finding among the neurons that we studied in area A1. Figure 7 plots two measures of spatial sensitivity for responses synchronized to a single sound source varying in location (horizontal axis) versus the same varying source in the presence of a competing sound fixed in location (vertical axis). The addition of a competing source consistently narrowed equivalent rectangular receptive fields (Fig. 7A) and deepened the range over which a neuron's spike rate varied as a function of sound-source location (modulation depth; Fig. 7B). This mutability in spatial sensitivity seemingly would aid in segregation of competing sound sequences while likely disrupting a labeled-line representation of source locations.

\section{Conclusion}

After 40 years of searching for a map of auditory space, I must concede failure to find such a map in the cat's auditory cortex. The cat is not alone in lacking a cortical space map. Studies of ferrets (e.g., King et al., 2007) and nonhuman primates (e.g., Woods et al., 2006; Remington and Wang, 2019) have shown a wide variety in the spatial tuning of cortical neurons, including very broad tuning, tuning that favors the frontal midline, and a remarkable percentage of neurons tuned to locations well above or below the horizontal plane or behind the animal. Nevertheless, those studies are unanimous in failing to show a spatial topography.

Neural topography of auditory space clearly is feasible. Auditory space maps in the avian optic tectum and mammalian superior colliculus coincide with topographic visual representations (barn owl: Knudsen, 1982; guinea pig: King and Palmer, 1983; cat: Middlebrooks and Knudsen, 1984; ferret: King and Hutching, 1987; monkey: Jay and Sparks, 1987). Those structures are important for directing head and eye movements to acoustic and other targets, and apparently those functions manifest as spatial topography. In contrast, the auditory cortex is essential for operant sound localization tasks, such as walking to or pointing at a remembered source location (Zatorre and Penhune, 2001; Malhotra et al., 2004). Those functions might be enhanced by a distributed representation that can adapt to varying task demands or ambient sounds. Notably, area anterior ectosylvian sulcus of the cat auditory cortex projects to the space-mapped superior colliculus (Meredith and Clemo, 1989), and auditory cortical projections in macaque translate to oculomotor coordinates in the posterior parietal cortex (Stricanne et al., 1996). Those observations indicate that non-space-mapped auditory cortex can conform to space-mapped coordinates when that is what is called for.

We have described a putative cortical substrate of one spatialhearing task, spatial stream segregation, which does not require explicit localization of sound sources, and there likely are others. Nevertheless, we cannot ignore the role of auditory cortex in overt localization tasks, and we would like to understand how that works. The absence of an auditory cortical space map precludes classes of experiments in which focal cortical stimulation might elicit a localized percept or movement or a focal inactivation might disrupt response to a sound in a particular region. I look forward to a new generation of approaches for probing a distributed code for sound-source locations. I would like to understand how the code is read out and integrated with other sensory and motor modalities for spatial perception and behavior.

Despite failing to find a space map in the auditory cortex, we have observed dynamic cortical mechanisms for spatial hearing that can adapt to a listener's needs for spatial hearing in a complex auditory scene. Single cortical neurons can signal the locations of sound sources panoramically, with small ensembles of as few as 16 neurons identifying sound-source locations with accuracy comparable to that of behaving cats. Spatial sensitivity of neurons is quite broad when an animal is idle, but spatial sensitivity sharpens rapidly when an animal is required to listen to sounds, particularly when it is required to localize. Confronted with sequences of sounds from sources separated by as little as $10^{\circ}$, a single neuron can synchronize selectively to one or the other of the sources. These mechanisms, even in a low-level cortical area, such as A1, provide us with valuable spatial functions for finding our way in the auditory world.

\section{References}

Briley PM, Kitterick PT, Summerfield AQ (2013) Evidence for opponent process analysis of sound source location in humans. J Assoc Res Otolaryngol 14:83-101.

Briley PM, Goman AM, Summerfield AQ (2016) Physiological evidence for a midline spatial channel in human auditory cortex. J Assoc Res Otolaryngol 17:331-340. 
Calford MB, Pettigrew JD (1984) Frequency dependence of directional amplification at the cat's pinna. Hear Res 14:13-19.

Dingle RN, Hall SE, Phillips DP (2010) A midline azimuthal channel in human spatial hearing. Hear Res 268:67-74.

Furukawa S, Xu L, Middlebrooks JC (2000) Coding of sound-source location by ensembles of cortical neurons. J Neurosci 20:1216-1228.

Imig TJ, Adrian HO (1977) Binaural columns in the primary field (A1) of the cat auditory cortex. Brain Res 138:241-257.

Imig TJ, Brugge JF (1978) Sources and terminations of callosal axons related to binaural and frequency maps in primary auditory cortex of the cat. J Comp Neurol 182:637-660.

Imig TJ, Irons WA, Samson FR (1990) Single-unit selectivity to azimuthal direction and sound pressure level of noise bursts in cat high-frequency primary auditory cortex. J Neurophysiol 63:1448-1466.

Javier LK, McGuire EA, Middlebrooks JC (2016) Spatial stream segregation by cats. J Assoc Res Otolaryngol 17:195-207.

Jay MF, Sparks DL (1987) Sensorimotor integration in the primate superior colliculus: II. Coordinates of auditory signals. J Neurophysiol 57:35-55.

Jenkins WM, Merzenich MM (1984) Role of cat primary auditory cortex for sound-localization behavior. J Neurophysiol 52:819-847.

King AJ, Hutching ME (1987) Spatial response properties of acoustically responsive neurons in the superior colliculus of the ferret: a map of auditory space. J Neurosci 57:596-624.

King AJ, Palmer AR (1983) Cells responsive to free-field auditory stimuli in guinea-pig superior colliculus: distribution and response properties. J Physiol 342:361-381.

King AJ, Bajo VM, Bizley JK, Campbell RA, Nodal FR, Schulz AL, Schnupp JW (2007) Physiological and behavioral studies of spatial coding in the auditory cortex. Hear Res 229:106-115.

Knudsen EI (1982) Auditory and visual maps of space in the optic tectum of the owl. J Neurosci 2:1177-1194.

Knudsen EI, Konishi M (1978) A neural map of auditory space in the owl. Science 2000:795-797.

Lee CC, Middlebrooks JC (2011) Auditory cortex spatial sensitivity sharpens during task performance. Nat Neurosci 14:108-114.

Lee CC, Middlebrooks JC (2013) Specialization for sound localization in fields A1, DZ, and PAF of cat auditory cortex. J Assoc Res Otolaryngol 14:61-82.

Makous JC, Middlebrooks JC (1990) Two-dimensional sound localization by human listeners. J Acoust Soc Am 87:2188-2200.

Malhotra S, Hall AJ, Lomber SG (2004) Cortical control of sound localization in the cat: unilateral cooling deactivation of 19 cerebral areas. J Neurophysiol 92:1625-1643.

Malhotra S, Stecker GC, Middlebrooks JC, Lomber S (2008) Sound localization deficits during reversible deactivation of primary auditory cortex and/or the dorsal zone. J Neurophysiol 99:1628-1642.

May BJ, Huang AY (1996) Sound orientation behavior in cats: I. Localization of broadband noise. J Acoust Soc Am 100:1059-1069.

Meredith MA, Clemo HR (1989) Auditory cortical projection from the anterior ectosylvian sulcus (field AES) to the superior colliculus in the cat: an anatomical and electrophysiological study. J Comp Neurol 289:687-707.

Merzenich MM, Knight PL, Roth GL (1975) Representation of cochlea within primary auditory cortex in the cat. J Neurophysiol 38:231-249.

Mickey BJ, Middlebrooks JC (2003) Representation of auditory space by cortical neurons in awake cats. J Neurosci 23:8649-8663.

Middlebrooks JC, Bremen P (2013) Spatial stream segregation by auditory cortical neurons. J Neurosci 33:10986-11001.

Middlebrooks JC, Green DM (1991) Sound localization by human listeners. Annu Rev Psychol 42:135-159.
Middlebrooks JC, Knudsen EI (1984) A neural code for auditory space in the cat's superior colliculus. J Neurosci 4:2621-2634.

Middlebrooks JC, Knudsen EI (1987) Changes in external ear position modify the spatial tuning of auditory units in the cat's superior colliculus. J Neurophysiol 57:672-687.

Middlebrooks JC, Onsan ZA (2012) Stream segregation with high spatial acuity. J Acoust Soc Am 132:3896-3911.

Middlebrooks JC, Pettigrew JD (1981) Functional classes of neurons in primary auditory cortex of the cat distinguished by sensitivity to sound location. J Neurosci 1:107-120.

Middlebrooks JC, Waters MF (2020) Spatial mechanisms for segregation of competing sounds, and a breakdown in spatial hearing. Front Neurosci 14:571095.

Middlebrooks JC, Dykes RW, Merzenich MM (1980) Binaural response-specific bands in primary auditory cortex (AI) of the cat: topographical organization orthogonal to isofrequency contours. Brain Res 181:31-48.

Middlebrooks JC, Clock AE, Xu L, Green DM (1994) A panoramic code for sound location by cortical neurons. Science 264:842-844.

Middlebrooks JC, Xu L, Eddins AC, Green DM (1998) Codes for soundsource location in nontonotopic auditory cortex. J Neurophysiol 80:863881.

Miller LM, Recanzone GH (2009) Populations of auditory cortical neurons can accurately encode acoustic space across stimulus intensity. Proc Natl Acad Sci USA 106:5931-5935.

Musicant AD, Chan JC, Hind JE (1990) Direction-dependent spectral properties of cat external ear: new data and cross-species comparisons. J Acoust Soc Am 87:757-781.

Phillips DP, Hall SE (2005) Psychophysical evidence for adaptation of central auditory processors for interaural differences in time and level. Hear Res 202:188-199.

Phillips DP, Calford MB, Pettigrew JD, Aitkin LM, Semple MN (1982) Directionality of sound pressure transformation at the cat's pinna. Hear Res 8:13-28.

Rajan R, Aitkin LM, Irvine DR, McKay J (1990a) Azimuthal sensitivity of neurons in primary auditory cortex of cats: I. Types of sensitivity and the effects of variations in stimulus parameters. J Neurophysiol 64:872-887.

Rajan R, Aitkin LM, Irvine DR (1990b) Azimuthal sensitivity of neurons in primary auditory cortex of cats: II. Organization along frequency-band strips. J Neurophysiol 64:888-902.

Remington ED, Wang X (2019) Neural representations of the full spatial field in auditory cortex of awake marmoset (Callithrix jacchus). Cereb Cortex 29:1199-1216.

Rice JJ, May BJ, Spirou GA, Young ED (1992) Pinna-based spectral cues for sound localization in cat. Hear Res 58:132-152.

Sabin AT, Macpherson EA, Middlebrooks JC (2005) Human sound localization at near-threshold levels. Hear Res 199:124-134.

Stecker GC, Harrington IA, Middlebrooks JC (2005) Location coding by opponent neural populations in the auditory cortex. PLoS Biol 3:e78.

Stricanne B, Andersen RA, Mazzoni P (1996) Eye-centered, head-centered, and intermediate coding of remembered sound locations in area LIP. J Neurophysiol 76:2071-2076.

Werner-Reiss U, Groh JM (2008) A rate code for sound azimuth in monkey auditory cortex: implications for human neuroimaging studies. J Neurosci 28:3747-3758.

Woods TM, Lopez SE, Long JH, Rahman JE, Recanzone GH (2006) Effects of stimulus azimuth and intensity on the single-neuron activity in the auditory cortex of the alert macaque monkey. J Neurophysiol 96:3323-3337.

Zatorre RJ, Penhune VB (2001) Spatial localization after excision of human auditory cortex. J Neurosci 21:6321-6328. 